\title{
A COMPARISON OF THE ELECTRICAL RESISTANCES OF THE SURFACE CELL MEMBRANE AND CELLULAR WALL IN THE PROXIMAL TUBULE OF THE NEWT KIDNEY
}

\author{
Takeshi HosHI AND Fuminori SAKAI \\ Department of Physiology and Department of Pharmacology, \\ Faculty of Medicine University of Tokyo, Tokyo
}

The proximal segment of the renal tubule is known to have relatively high permeabilities to electrolytes and non-electrolytes of small molecular sizes. Considerable inward movements of radioactive sodium and potassium were shown to occur in frog kidney when these isotopic ions were injected into the renal portal vein ${ }^{1)}$. Similar inward fluxes of sodium, urea, glycerol and other non-electrolytes were shown also in mammalian kidneys under stop-flow conditions ${ }^{23)}$. According to WILDE and MALVIN ${ }^{2)}, \mathrm{Na}^{24}$ leaks very fast into the proximal segment, where it equilibrates with plasma within two to four minutes after intravenous injection. Measurements of unidirectional fluxes of sodium by OKEN et al.4) and of chloride by $\mathrm{GIEBISCH}^{5)}$ revealed that very rapid turnover of these ions was taking place across the proximal tubule. Thus, the tubular wall of this segment has frequently been referred to as leaky sieve ${ }^{3,4)}$, and this leaky property has been considered to be responsible for the characteristic mode of solute transfer in this segment, viz., the gradient limiting reabsorption of sodium $^{6)}$ and isotonic reabsorption of various electrolytes ${ }^{7}$.

The aim of this study was to see whether the leaky property of the proximal segment is due to high permeability of the plasma membrane of the tubular cells or due to the presence of some extracellular shunt paths within the tubular wall through which small solutes can pass without entering the cells. Our preliminary study, in which double insertion of microelectrodes was carried out and a minute electric current was passed through one electrode and resultant potential changes were recorded with the other electrode, disclosed that the wall of this segment behaved like a core conductor. This observation led us to an attempt to measure the specific resistance of the surface cell membrane and to compare it with that of the cellular wall. The results show that the specific resistance of the surface membrane is the same order as that

Received for publication February 9, 1967

星 猛, 酒井文徳 
of a wide variety of excitable cells, while the resistance of the cellular wall is much lower than the value expected from the resistance of the surface membrane. This strongly suggests that the lumen is not completely covered by the surface membrane; in other words, there exist vast extracellular shunt paths for electrolytes within the wall of the tubules. A preliminary report of this study was published elsewhere ${ }^{8)}$.

\section{METHODS}

Materials: Male newt (Triturus pyrrhogaster) were used throughout experiments. The animals were pithed and their abdominal wall was widely opened to expose unilateral kidney. Newt kidney consists of two distinct parts, one the sexual kidney and the other the definitive kidney (see reference ${ }^{9}$ ). Both parts were subjected to experiments equally. Identification of the proximal segment was made by measuring the outer diameter of the tubule under a stereomicroscope since a preliminary histological study revealed that the proximal segment had a relatively large outer diameter more than $80 \mu$.

After having been exposed the kidney, whole body of the animal was fixed on a cork board attached to the bottom of a small lucite chamber filled with oxygenated Ringer solution. The animal was washed several times with fresh Ringer solution before starting experiments. Ringer's solution used had following composition (in $\mathrm{mM}$ ) : $\mathrm{NaCl} ; 105, \mathrm{KCl} ; 2.65, \mathrm{CaCl}_{2} ; 1.8, \mathrm{Na}_{2} \mathrm{HPO}_{4} ; 2.25, \mathrm{NaH}_{2} \mathrm{PO}_{4} ; 0.4$, Glucose ; 5.5 (pH 7.2). All experiments were carried out during winter and early spring at room temperature $\left(16-20^{\circ} \mathrm{C}\right)$.

Recording of transmembrane and transtubular potentials: Ling-Gerard type glass microelectrodes filled with $3 \mathrm{M} \mathrm{KCl}$ were used for recording both transmembrane and transtubular potentials. The electrodes were filled with $\mathrm{KCl}$ solution directly with the aid of a fine metal needle and only those of a moderately high resistance, ranging from 15 to $25 \mathrm{M} \Omega$ were used. A coiled $\mathrm{AgAgCl}$ wire was used as a reference electrode which was immersed in the bathing fluid. The potential led out was amplified by a cathodefollower preamplifier and then displayed on a screen of a double-beam oscilloscope, Du Mont type-322A.

Application of electric current: Electric current was applied through another microelectrode inserted into a cell or a lumen by connecting the electrode to a square-pulse generator via $50 \mathrm{M} \Omega$. The current actually passed was recorded by amplifying the potential drop across a $10 \mathrm{~K} \Omega$ resistor connected between the reference electrode and the ground. This also was displayed on the same oscilloscope.

Localization of electrode tips: One of difficulties in the present experiments was the assurance of the correct position of electrode tips. In the case of double insertion into the cell inside, this was not so serious, but, in the case of intraluminal double insertion, it was much difficult. Sometimes, the tips were seen in the lumen under a microscope, but this was not frequent. A practically easier way for the latter case was to observe the potential steps according to advancement and withdrawal of the electrode. When the tip penetrated the peritubular membrane, an abrupt potential drop, by about $70 \mathrm{mV}$, was recorded as described in a previous paper ${ }^{10)}$. Further advancement of the tip caused a sudden but irregular shift of the potential toward zero level and then the potential dropped again to nearly the same level as obtained initially. This second deep stable negativity was obtained when the tip penetrated the luminal membrane of the cell on the opposite side. The electrode was then gently pulled back 
until a sudden shift of the potential toward zero level was recorded. The small negativity thus obtained was quite stable and varied in a narrow range from 0 to $-20 \mathrm{mV}$. This stable small negativity was regarded as the true transtubular potential and an indication of intraluminally located electrode tip.

\section{RESULTS}

1. Responses of transmembrane potential to applied current. Fig. 1 shows the typical changes in the transmembrane potential on the peritubular side of the proximal tubular cell produced by the intracellular application of a square-wave current pulse. In this figure, a current pulse of $6 \times 10^{-7} \mathrm{~A}$ and $50 \mathrm{msec}$ duration was applied and resultant potential changes were recorded from different distances from the site of the current electrode. The shape of the potential change was essentially the same as that of the electrotonic potential seen in nerve and muscle fibers under similar experimental conditions. The time

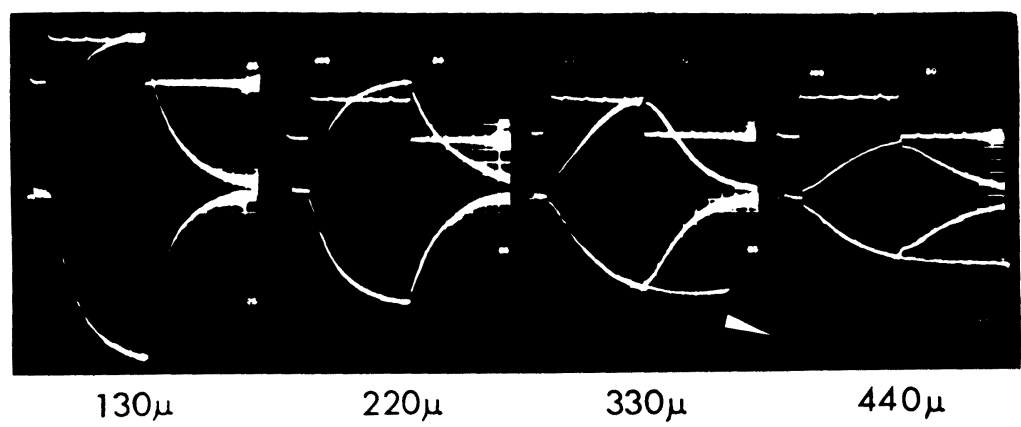

Fic. 1. The changes in the transmembrane potential of the proximal tubular cells of the newt kidney caused by the intracellular application of electric current of $6 \times 10^{-7} \mathrm{~A}$ and $50 \mathrm{msec}$ duration. Two sweeps were superposed, one for the response to the depolarizing current and the other to the hyperpolarizing current. Only the depolarizing current was displayed by using another beam (uppermost tracings), small notches on which are the time marks of $10 \mathrm{msec}$. Interelectrode distance was shown below each set of tracings.

course of the initial phase of the change was nearly an exponential function of time and the potential reached its maximum level in about $50 \mathrm{msec}$ in the vicinity of the current electrode. The maximum deflection was linearly proportional to the strength of applied current within the range up to $10^{-6} \mathrm{~A}$. There was no difference both in the time course and the amplitude of the deflection between for the outward (depolarizing) and the inward (hyperpolarizing) currents. No particular self-generative response was seen even when the cell inside was made positive relative to the outside medium (FIG. 1,a). Only in a small number of nephrons in the upper part of the definitive kidney, the initial segment of which was abnormally ampulated, cells showed 
some rectifying property, i. e., a greater deflection for the outward current than for the inward current, but this phenomenon was rather exceptional. Generally, the surface membrane of the proximal tubular cells behaved like a simple fixed resister over a wide range of current strength.

It was a rather surprising observation that this type of change in the transmembrane potential was recorded, without exception, from cells considerably distant from the site of the current application. As seen in FIG. 1, even a cell about a half $\mathrm{mm}$ apart from the cell impaled with the current electrode still showed the potential change of a considerable size. This means that there is a significant intracellular longitudinal current spread under these conditions and the current reaches quite distant cells beyond many intercellular boundaries. The decay of the potential change was found to be an exponential function of the interelectrode distance as shown in FIG. 2. Although the

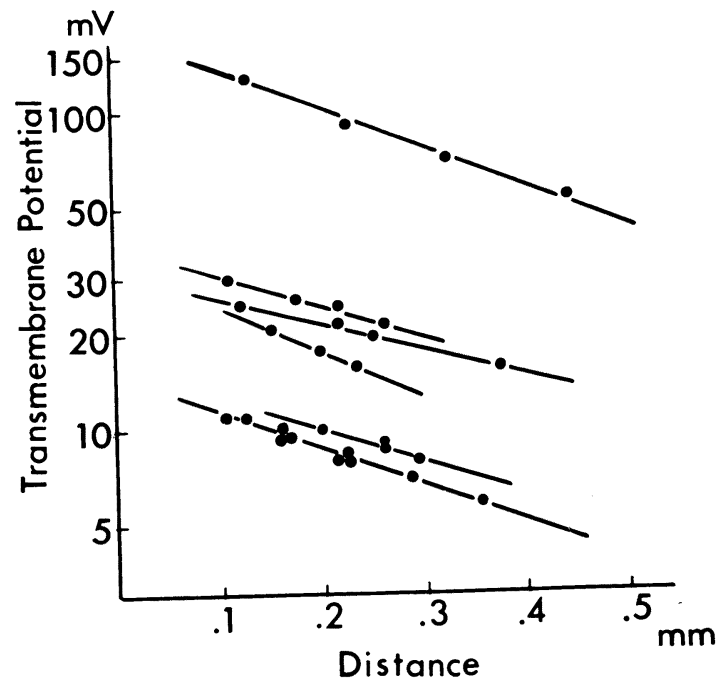

FIG. 2. The spatial decay of the transmembrane potential changes caused by the intracellularly applied current. Data from six different proximal tubule.

interelectrode distance examined in the present experiments was limited to the range from 100 to $500 \mu$, the exponential equation for the spatial decay of the passive electrotonic potential in a simple core conductor ${ }^{11,2)}$ was applied to the present case, and the space constant $(\lambda)$ and the effective resistance $\left(2 E_{0} / I\right)$ were estimated. The average values for $\lambda$ and $2 E_{0} / I$ were $400 \mu$ and $3.1 \times 10^{5} \Omega$, respectively, (TABLE 1). It should be pointed out that these values were dependent on the spontaneous transmembrane potential of the tubular celis. As shown in Fig. 3, definite tendency was observed that the smaller the transmembrane potential, the higher the effective resistance. 
TABLE 1.

Dimension of the proximal tubules of the newt kidney and estimated values for the space constant and the effective resistance of the nephron wall.

\begin{tabular}{c|c|c|c|c}
$\begin{array}{c}\text { Tubule } \\
\text { Number }\end{array}$ & $\begin{array}{c}\text { Outer } \\
\text { Diameter } \\
\mu\end{array}$ & $\begin{array}{c}\text { Wall } \\
\text { Thickness } \\
\mu\end{array}$ & $\begin{array}{c}\lambda \\
\mathrm{mm}\end{array}$ & $\begin{array}{c}2 E_{0} / I \\
\times 10^{5} \Omega\end{array}$ \\
\hline 1 & 130 & 20 & 0.35 & 7.2 \\
2 & 100 & 15 & 0.18 & 3.0 \\
3 & 140 & 30 & 0.44 & 1.3 \\
4 & 140 & 30 & 0.44 & 1.3 \\
5 & 140 & 30 & 0.40 & 1.5 \\
6 & 130 & 25 & 0.58 & 4.2 \\
Mean & 130 & 25 & 0.40 & 3.1 \\
\hline
\end{tabular}

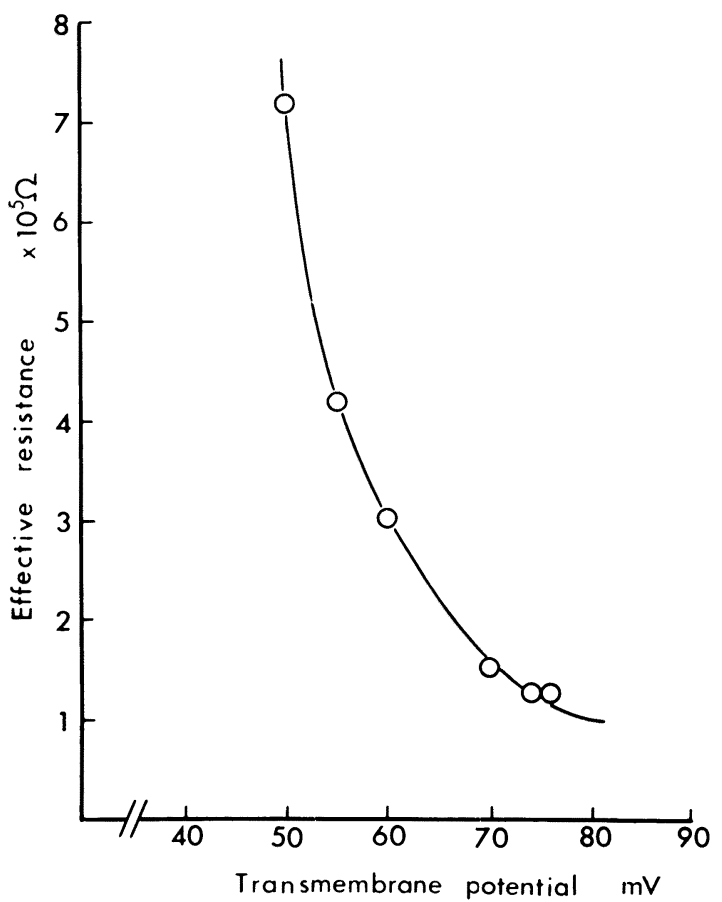

FIG. 3. Relationship between the effective resistance of the tubular wall, as determined by double intracellular insertion of microelectrodes, and the transmembrane potential of the cells of the same tubules.

2. Specific resistance of the surface membrane. Above findings indicate that the surface cell membrane of the proximal tubule has relatively poor conductivity while the intercellular membranes are relatively highly conductive. Thus, the situation of the tubular wall resembles that of a core conductor. 


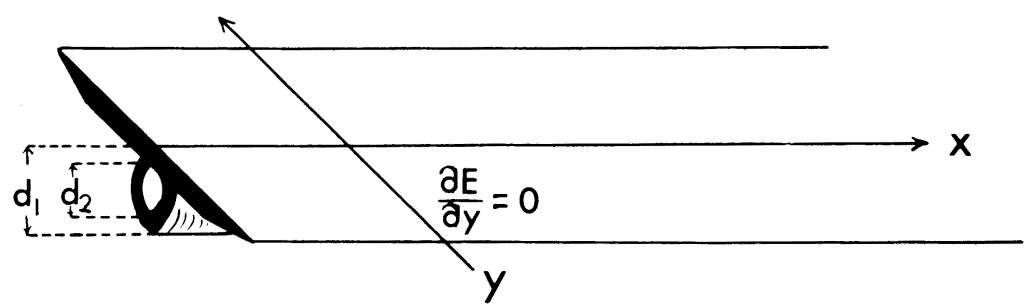

FIG. 4. A simplified core conductor model applied to the proximal tubular wall.

This led us to a calculation of the specific resistances for the surface membrane $(R m)$ and cytoplasm including intercellular membranes $(R i)$ by applying the core conductor theory ${ }^{11,12)}$. For the sake of simplicity, the tubular wall was assumed to be a sufficiently thin and long plate conductor as shown in FIG. 4, and the simple cable theory was applied to this model. Both sides of the plate were assumed to be insulated. In this model $R m$ and $R i$ are given by following equations :

$$
\begin{aligned}
& R m=r_{m} \cdot \pi \cdot\left(d_{1}+d_{2}\right) \\
& R i=r_{i} \cdot \frac{\pi}{4}\left(d_{1}^{2}-d_{2}{ }^{2}\right)
\end{aligned}
$$

where $r_{m}$ is the resistance of the surface membrane multiplied by unit length of the membrane, $r_{i}$ is the resistance of the core per unit length, $d_{1}$ and $d_{2}$ are outer and inner diameters of the tubule. $r_{m}$ and $r_{i}$ were calculated from the values for $\lambda$ and $2 E_{0} / I$ above described. Average values for $R m$ and $R i$ thus obtained were $836 \Omega \mathrm{cm}^{2}$ and $625 \Omega \mathrm{cm}$, respectively.

3. Transtubular (cell wall) resistance and its comparison with the surface membrane resistance. When a current pulse of order of $10^{-7} \mathrm{~A}$ was applied through a microelectrode inserted into the lumen, the change in the transtubular potential was so small even in the vicinity of the current electrode, that an accurate estimation of the space constant was extremely difficult. Application of a stronger current, greater than $10^{-6} \mathrm{~A}$, was possible in a few experiments, but even in such cases, the potential decayed sharply around the site of the application of current.

If the tubular lumen is completely covered with the double surface membrane (peritubular and luminal membranes), such a sharp decay of the passive transtubular potential can hardly be interpreted. If we assume that the tubule is a cylindrical core conductor as a whole in which the luminal fluid (core) having nearly the same specific resistance as that of Ringer solution is completely covered with double surface membrane, it becomes clear that the values of $836 \Omega \mathrm{cm}^{2}$ for the surface membrane and of $80 \mu$ for the inner diameter give a much longer space constant and a moderately high effective resistance, namely, $2.5 \mathrm{~mm}$ and $2.5 \times 10^{5} \Omega$, respectively. These values imply that if the 
changes in the transtubular potential caused by the intraluminal application of current was compared with those in the transmembrane potential caused by the intracellular application of current of the same strength in the same nephron, the size of the potential change would be about the same in the

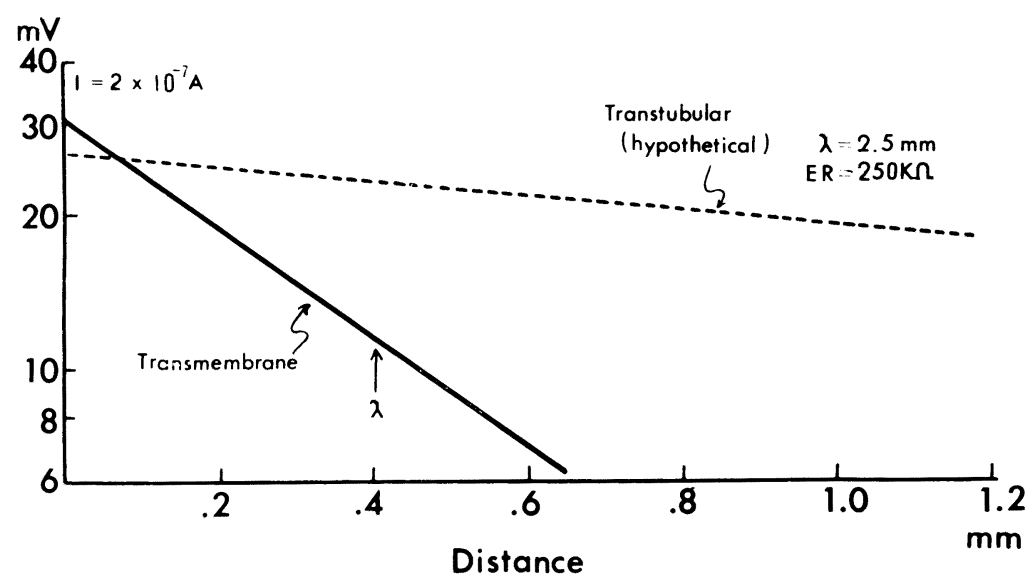

Fig. 5. Comparison of the spatial decay of the passive transmembrane and transtubular potentials in the same nephron. The decay of the passive transmembrane potential (heavy line) was calculated by using the estimated mean values for $\lambda(400 \mu)$ and the effective resistance $\left(3.1 \times 10^{-5} \Omega\right)$, and an arbitrary chosen current strength of $2 \times 10^{-7} \mathrm{~A}$. The decay of the passive transtubular potential (dotted line) was calculated on the assumption that the lumen of $80 \%$ diameter was completely covered by double membrane, of which $R m$ was $836 \Omega \mathrm{cm}^{2}$, and by using the same current strength as in the former case.

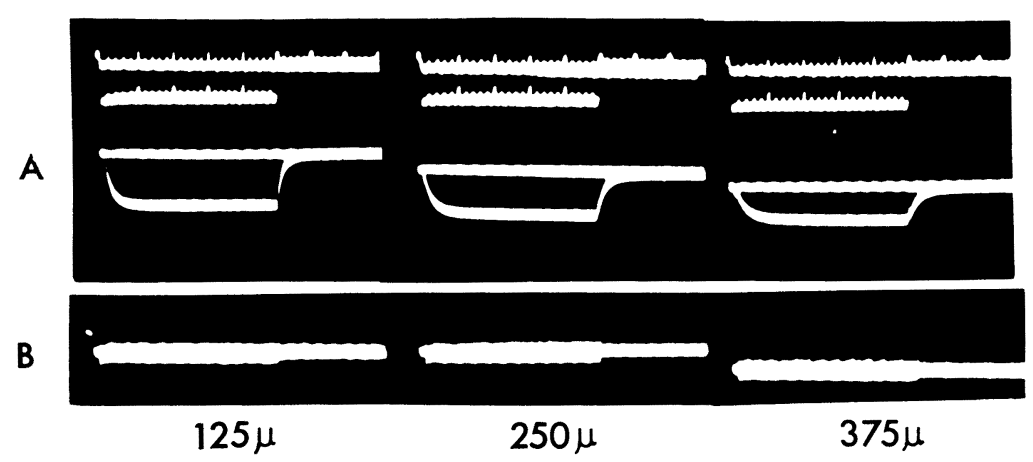

FIG. 6. Actual tracings of the passive transmembrane potential change caused by the intracellularly applied current of $2 \times 10^{-7} \mathrm{~A}$ (A) and of the passive transtubular potential change caused by the intraluminally applied current of the same strength (B). The uppermost tracings show the current pulses actually passed. Time marks, superposed on the current tracings, are 10 and $50 \mathrm{msec}$. Interelectrode distance was shown below each tracings. 
vicinity of the current electrode in both cases, but the changes in the transtubular potential should be much larger at distant points as shown in the FIG. 5. Actual comparison of this type was carried out with the same current strength, but, as shown in FIG. 6 , the results were the reverse. The change in the transtubular potential was much smaller than in the transmembrane potential over the whole range of the interelectrode distance examined. This fact clearly indicates that the lumen is not completely covered with the surface membrane.

\section{DISCUSSION}

The value of $R m$ for the surface membrane obtained in the present study is not far different from those of the plasma membrane of some types of excitable cells, for example, nonmyelinated nerve fiber of squid $\left(1000 \Omega \mathrm{cm}^{2}\right)^{13}$ and Purkinje fibers of sheep $\left(1200 \Omega \mathrm{cm}^{2}\right)^{14}$. However, the cells of the proximal tubule of vertebrate kidneys are known to have microvilli on the apical side and many membrane infoldings on the opposite side ${ }^{15)}$. It may, therefore, be reasonable to think that the real specific resistance of the surface membrane must be much higher than the value described above. No correction was made for such processes and infoldings because no information was available concerning the quantitative aspects of the membrane structure of the tubular cells in the newt. If allowance is made for such membrane structure, one should consider that the surface membrane of the proximal tubule is rather poorly conductive as compared with cell membranes of a wide variety of cells. The value for $R i$, on the other hand, is several times as high as that of myoplasm of amphibian skeletal muscle $(250 \Omega \mathrm{cm})^{12}$. As the specific resistance of cytoplasm may not significantly differ among different types of cells, the difference should be ascribed to the presence of the intercellular membranes or incomplete (partial) fusion of cells. Any way, the fact that the potential change caused by the intracellular application of current reaches distant cells beyond many intercellular boundaries suggests that the cells are tightly attached, at least in part, and the junction of such parts is leaky. Similar property of the intercellular junction has recently been demonstrated in some other epithelial tissues ${ }^{16}$.

In the present study, the tubular wall was assumed to be a sufficiently thin and long plate conductor and the simple cable theory was applied to this model. There may be some errors in the estimated values due to such simplification. One of sources of errors may be a radial current flow in the vicinity of the current electrode. According to TAYLER ${ }^{17)}$, however, the error of $\lambda$, due to application of the simple cable theory to a model with a radial current flow, is insignificant if the ratio of $\lambda$ to the diameter of a cylindrical conductor is sufficiently large, viz., more than 1 . In our model, $\lambda / d_{1}$ was greater than 6 , 
and when the plate model is converted into a cylinder with the same crosssectional area as that of the original plate, the ratio of $\lambda$ to the equivalent diameter becomes 12.5. Accordingly, the error of $\lambda$ may not be significant. Rather important factor which affects the values of $\lambda$ and $2 E_{0} / I$ was the transmembrance potential of the cells. In the present experiments, the peritubular transmembrane potential varied from 50 to $75 \mathrm{mV}(64 \mathrm{mV}$ on the average), which corresponds to the normal variation in the newt tubule ${ }^{10}$. Recently, a quite similar value of $\lambda$ was obtained by WINDHAGER et al. ${ }^{18}$ in proximal tubule of the newt kidney.

Although it was not possible to estimate the space constant for the passive transtubular potential, this seemed to be far less than $100 \mu$ in the present study. WINDHAGER and GIEBISCH ${ }^{19)}$ carried out a similar experiment in the proximal tubule of rat kidney, and obtained $55 \mu$ for the space constant. Their experimental conditions were somewhat different from ours, for example, the luminal fluid was separated by oil columns in their experiments. The inner diameter of the rat tubule is significantly smaller than that of the triturus, but their value and our findings suggest that the wall of the proximal tubule is quite leaky irrespective of animal species. Such short space constant can not be accounted for by the surface membrane resistance since a simple calculation based on the core conductor theory gives much longer value provided that the lumen is completely covered with the surface membrane. Therefore, it is quite probable that there are abundant extracellular shunt paths within the tubular wall. Recently, WINDHAGER and GIEBISCH ${ }^{18)}$ also reached the same conclusion from their study in Necturus kidney and, in addition, they demonstrated that this shunt could be modified by changing the tonicity of the outside medium. Whether such shunt penetrates the cell body or locates. between cells is not known at present.

\section{SUMMARY}

1. The electrical properties of the proximal tubular cells were studied in the newt kidney by using microelectrode techniques.

2. The surface membrane of the tubular cells behaved like a simple resistor when a minute current was applied intracellularly. The changes in the transmembrane potential was linearly proportional to the strength of applied current and there was neither the rectification nor the self-generative response within a wide range of current strength.

3. The potential change caused by the intracellular application of current spread over considerable distances along the longitudinal axis of the tubule. The tubular wall, thus, behaved like a core conductor.

4. Application of the simple core conductor theory to the model of the tubular wall gave the values of $400 \mu$ for the space constant, $3.1 \times 10^{5} \Omega$ for the effective 
resistance, $836 \Omega \mathrm{cm}^{2}$ for the specific resistance of the surface membrane and $625 \Omega \mathrm{cm}$ for the specific resistance of the core (cytoplasm including intercellular membranes).

5. A comparison of the surface membrane resistance to the transtubular resistance suggests that there are significant extracellular shunt paths for electrolytes within the tubular wall. The leaky nature of the proximal segment was ascribed to the presence of such shunt paths.

The authors wish to thank Professor K. MAtsuda for his support and reviewing the manuscript, Drs. G. Giebisch and E.E. Windhager for their useful discussions and Dr. J. W. Moore for his valuable criticism. Thanks are also due to Dr. M. HAGA for his assistance during experiments.

\section{REFERENCES}

1) Hoshiko, T., Swanson, R. E. And Visscher, M. B.: Excretion of $\mathrm{Na}^{22}$ and $\mathrm{K}^{42}$ by the perfused bullfrog kidney and the effect of some poisons. Amer. J. Physiol. 184: 542,1959

2) Wilde, W.S. ANd MALvin, R. L.: Movement of peritubular Na ${ }^{24}$ across the nephron wall during stop-flow. Fed. Proc. $18: 170,1959$.

3) PÊNa, J.C. ANd MALvin, R.L.: Transcellular diffusion of non-electrolytes across the renal tubular epithelium. J. Gen. Physiol. 45: 643, 1962.

4) Oken, D.E., Whittembury, G., Windhager, E. E., Schatzmanin, H. J. and Solomon, A.K.: Active transport of sodium by the proximal tubule of Necturus. J. Clin. Invest. $38: 1029,1959$.

5) Giebisch, G.: Measurements of electrical potentials and ion fluxes on single renal tubules. Nierensymposion, Georg Thieme, Stuttgart, 1960, p. 49.

6) Windhager, E.E., Whittembury, G., Oken, D. E., Schatzmann, H. J. ANd Solomon, A.K.: Single proximal tubules of the Necturus kidney. III. Dependence of Water movements on $\mathrm{NaCl}$ concentration. Amer. J. Physiol. $197: 313,1959$.

7) Giebisch, G. And Windhager, E.E.: Renal tubular transfer of sodium, chloride and potassium. Amer. J. Med. 36: 643, 1964.

8) Hoshi, T., Sakai, F. And HaGa, M.: Electrical properties of renal tubules of the newt. J. Physiol. Soc. Japan, $24: 378,1962$.

9) Francis, E.T.B.: The anatomy of the salamander. Oxford, Clarendon Press, 1934.

10) Sakai, F., Hoshi, T., Haga, M. and Enomoto, Y.: Membranpotential an der Nierentubuli des Triturus pyrrhogaster. Jap. J. Pharmacol. 11:65, 1961.

11) Hodgkin, A.L. And Rushton, W. A.H.: The electrical constants of a crustacean nerve fibre. Proc. Roy. Soc. London, B. $133:$ 444, 1946.

12) Fatt, P. And Katz, B.: An analysis of the end-plate potential recorded with an intracellular electrode. J. Physiol. 115: 320, 1951.

13) Cole, K.S. And Hodgkin, A.L.: Membrane and protoplasm resistance in the squid giant axon. J. Gen. Physiol. 22 : 671, 1939.

14) Weidmann, S.: Elektrophysiologie der Herzmuskelfaser. Hans Huber, Bern, 1957.

15) Roberts, J.S. And Schmidt-Nielsen, B.: Renal ultrastructure and excretion of salt and water by three terrestrial lizards. Amer. J. Physiol. 211: 476, 1966.

16) Lowenstein, W.R.: Permeability of membrane junctions. Ann. N. Y. Acad. Sci., $137: 441,1966$. 
17) TAYLOR, R.E.: Cable theory. Physical techniques in biological research. Edited by W.L. Nastuk, Vol. VI. Electrophysiological methods. Part B. Academic Press, New York, 1963, Page 219.

18) Windhager, E.E., Klose, R. M. and Giebisch, G.: Measurements of electrical conductances in single proximal tubules of Necturus kidney. XXIII Internat. Congr. of Physiol. Sci., Tokyo, 1965, Abstract p. 165.

19) Windhager, E. E. And Giebisch, G.: Comparisons of short-circuit current and net water movement in single perfused proximal tubules of rat kidney. Nature, 191: (4794) p. $1205,1961$. 